\section{Ehrlichia muris in Ixodes cookei Ticks, Northeastern United States, 2016-2017}

\section{Guang Xu, Patrick Pearson, Stephen M. Rich}

Author affiliation: University of Massachusetts-Amherst, Amherst, Massachusetts, USA

DOI: https://doi.org/10.3201/eid2406.171755

Ehrlichia muris is an agent of human ehrlichiosis. To determine its geographic spread in the United States, during 2016-2017, we tested 8,760 ticks from 45 states. A distinct clade of $E$. muris found in 3 Ixodes cookei ticks from the northeastern United States suggests transmission by these ticks in this region.

E hrlichia muris was originally isolated from a mouse in Japan in 1983 (1). In 2009 in the United States, an E. muris-like agent (EMLA) was identified as a causative agent of human ehrlichiosis for 3 symptomatic patients in Wisconsin and 1 in Minnesota (2). A retroanalysis of 760 Ixodes scapularis ticks collected from 1992 through 1997 in Wisconsin revealed an EMLA infection rate of 0.94\%, indicating presence of this pathogen in the upper midwestern region since at least the mid-1990s (3). Another study found this infection in 69 patients from 5 states from 2007 through 2013, although all patients had probably been exposed to the ticks in Minnesota or Wisconsin (4). In 2017, the E. muris-like isolates from the upper midwestern United States were proposed as a taxonomically distinct subspecies, E. muris subsp. eauclairensis (5).

E. muris is thought to be transmitted by Haemaphysalis flava ticks in Japan, by I. persulcatus ticks in eastern Europe, and by I. ricinus ticks in western Europe (5). Detection of the bacterium in nymphal and adult stages of I. scapularis ticks $(2,5,6)$ and in white-footed mice (Peromyscus leucopus) suggests that the primary vectors and reservoir hosts of Lyme borreliosis play a major role in the enzootic transmission cycle of E. muris in the United States. However, despite the broad distribution of I. scapularis ticks and P. leucopus mice in North America, to our knowledge, E. muris has not been reported outside of Wisconsin and Minnesota $(2,7)$.

To evaluate the geographic distribution of E. muris from May 30, 2016, through October 1, 2017, we used a Taqman real-time PCR to test 8,760 ticks for EMLA, Anaplasma phagocytophilum, Borrelia burgdorferi sensu lato, B. miyamotoi, B. mayonii, and Babesia microti. The EMLA test is a modified version of a multiplex Taqman assay and targets the p13 gene (8). The human-biting ticks used for this study were submitted to the TickReport public testing program (https://www.tickreport.com) at the University of Massachusetts (Amherst, MA, USA). We confirmed EMLA positivity of the samples by amplifying and sequencing the EMLA citrate synthase ( $g l t A)$ and heat shock protein (gro$E L$ ) genes (3). We confirmed the species of EMLA-positive ticks by amplifying and sequencing a partial tick $16 \mathrm{~S}$ rRNA gene (online Technical Appendix, https://wwwnc.cdc.gov/ EID/article/24/6/17-1755-Techapp1.pdf). We received 8,760 ticks from 45 states: 243 Amblyomma americanum, 2 A. maculatum, 7 Amblyomma spp., 6 Dermacentor andersoni, 3 D. occidentalis, 271 D. variabilis, 45 Dermacentor spp., 14 I. angustus, 22 I. cookei, 215 I. pacificus, 5 I. ricinus, 7,800 I scapularis, 19 I. spinipalpis, 47 Ixodes spp., and 7 Rhipicephalus sanguineus; 54 ticks were unidentifiable.

We found DNA specific for EMLA in only 2 species of Ixodes tick: I. scapularis and I. cookei. The overall prevalence of EMLA was very low. Only $5(0.057 \%)$ ticks were positive for E. muris-specific DNA. Although we tested 7,800 I. scapularis ticks from 33 states in the northeastern, midwestern, and southeastern regions, we found only $2(2 / 7,800,0.026 \%)$ EMLA-positive $I$. scapularis ticks (1 from Laporte, MN, and 1 from Eleva, WI). These 2 ticks were co-infected with $B$. burgdorferi s. 1. and B. microti. However, no DNA from B. miyamotoi, B. mayonii, or $A$. phagocytophilum was detected in these 2 ticks.

The prevalence of EMLA in I. cookei ticks was much higher than that in I. scapularis ticks. Of the 22 I. cookei ticks tested, $3(3 / 22,13.64 \%)$ were positive for EMLA (from Holden, ME; Littleton, ME; and Salamanca, NY). Co-infections were not detected in these 3 ticks.

To determine the identity of these EMLA isolates, we examined gltA and groEL gene sequences of isolates from the 2 I. scapularis ticks and found them to be identical. Phylogenetic analysis showed that they clustered with $E$. muris subsp. eauclairensis. The gltA and groEL gene sequences from the $3 I$. cookei ticks were also identical but formed a new clade between E. muris subsp. eauclairensis and subsp. muris (Figure).

The detection of E. muris in I. scapularis ticks from the upper midwestern United States corroborates previously reported findings $(2,3,6)$. The detection of a distinct clade of E. muris in I. cookei ticks from the northeastern United States represents a potential risk to humans or a different enzootic cycle of E. muris in the Northeast. As primary vectors of Powassan virus (lineage 1), I. cookei ticks are widely distributed in eastern North America and are the second most common species of Ixodes ticks found on persons in Maine, USA (9). Further study is warranted with regard to the vector competence of $I$. cookei ticks for transmitting E. muris, the natural enzootic cycle of E. muris, and the transmission potential of the pathogen to humans in this region. Meanwhile, human ehrlichiosis should be considered as a possible diagnosis for persons who have been 


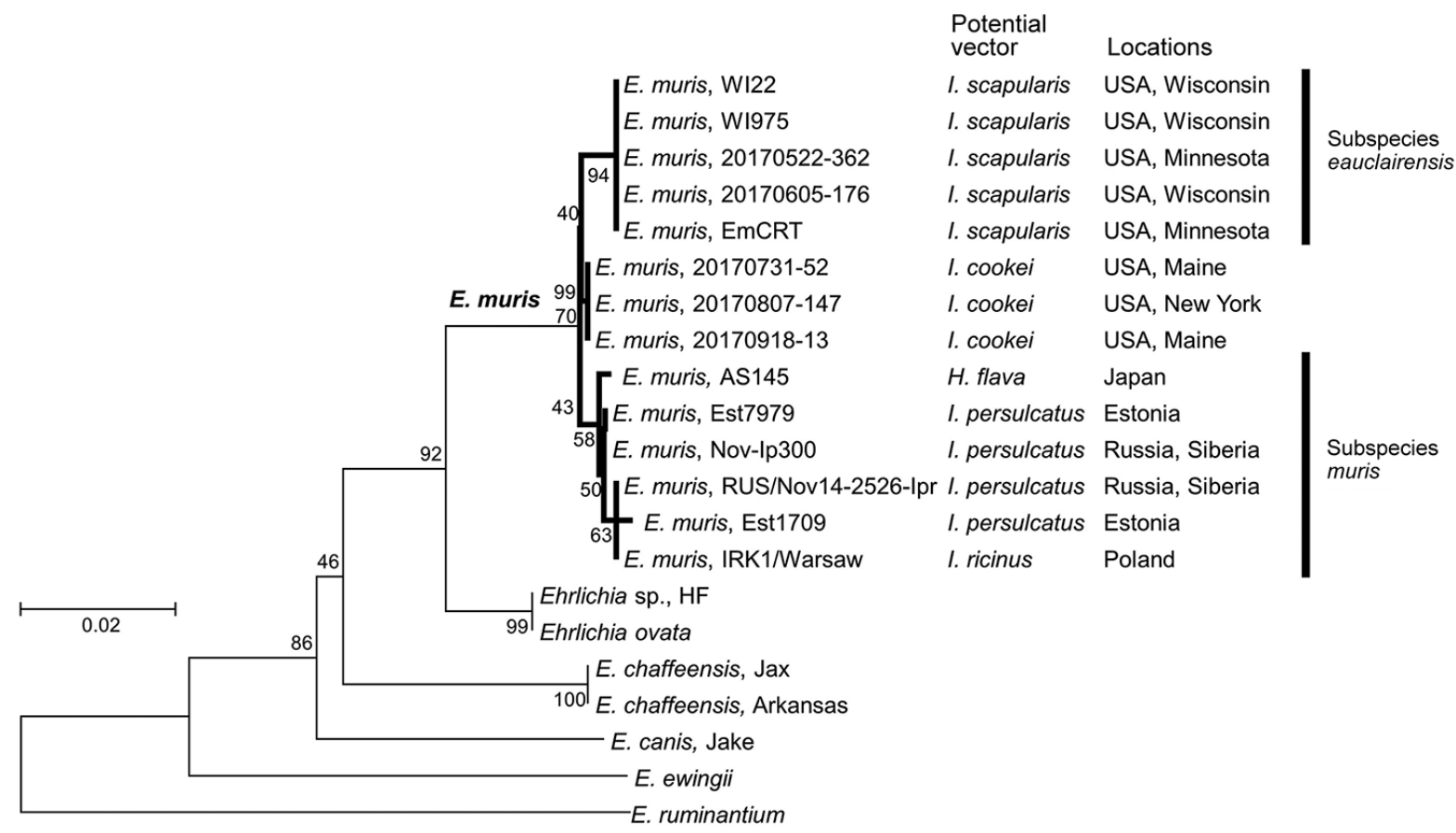

Figure. Phylogenetic tree of Ehrlichia citrate synthase ( $g l t A)$ and heat shock protein ( $g r o E L)$ genes constructed by the maximumlikelihood method of MEGA6 software (http://www.megasoftware.net). The total length of 2 concatenated genes is 1,045 bp. HasegawaKishino-Yano with invariable sites was selected as the best model based on Bayesian information criterion scores. Numbers on the branches represent bootstrap support with 500 bootstrap replicates. Scale bar indicates nucleotide substitutions per site.

exposed to I. scapularis and I. cookei ticks in the upper midwestern and northeastern United States, respectively.

\section{Acknowledgment}

We thank Timothy Daly and Fumiko Ribbe for sample preparation and DNA extractions.

\section{About the Author}

Dr. Xu is an extension associate professor in the Laboratory of Medical Zoology, Department of Microbiology, University of Massachusetts-Amherst. His research interests include tick and tickborne diseases.

\section{References}

1. Wen B, Rikihisa Y, Mott J, Fuerst PA, Kawahara M, Suto C. Ehrlichia muris sp. nov., identified on the basis of $16 \mathrm{~S}$ rRNA base sequences and serological, morphological, and biological characteristics. Int J Syst Bacteriol. 1995;45:250-4. http://dx.doi.org/10.1099/00207713-45-2-250

2. Pritt BS, Sloan LM, Johnson DKH, Munderloh UG, Paskewitz SM, McElroy KM, et al. Emergence of a new pathogenic Ehrlichia species, Wisconsin and Minnesota, 2009. N Engl J Med. 2011;365:422-9. http://dx.doi.org/10.1056/NEJMoa1010493

3. Telford Iii SR, Goethert HK, Cunningham JA. Prevalence of Ehrlichia muris in Wisconsin deer ticks collected during the mid 1990s. Open Microbiol J. 2011;5:18-20. http://dx.doi.org/ 10.2174/1874285801105010018

4. Johnson DKH, Schiffman EK, Davis JP, Neitzel DF, Sloan LM, Nicholson WL, et al. Human infection with Ehrlichia muris-like pathogen, United States, 2007-2013. Emerg Infect Dis. 2015;21:1794-9. http://dx.doi.org/10.3201/eid2110.150143

5. Pritt BS, Allerdice MEJ, Sloan LM, Paddock CD, Munderloh UG, Rikihisa Y, et al. Proposal to reclassify Ehrlichia muris as Ehrlichia muris subsp. muris subsp. nov. and description of Ehrlichia muris subsp. eauclairensis subsp. nov., a newly recognized tick-borne pathogen of humans. Int J Syst Evol Microbiol. 2017;67:2121-6. http://dx.doi.org/10.1099/ijsem.0.001896

6. Stromdahl E, Hamer S, Jenkins S, Sloan L, Williamson P, Foster E, et al. Comparison of phenology and pathogen prevalence, including infection with the Ehrlichia muris-like (EML) agent, of Ixodes scapularis removed from soldiers in the midwestern and the northeastern United States over a 15 year period (1997-2012). Parasit Vectors. 2014;7:553. http://dx.doi.org/10.1186/s13071-014-0553-Z

7. Wormser GP, Pritt B. Update and commentary on four emerging tick-borne infections: Ehrlichia muris-like agent, Borrelia miyamotoi, deer tick virus, Heartland virus, and whether ticks play a role in transmission of Bartonella henselae. Infect Dis Clin North Am. 2015;29:371-81. http://dx.doi.org/10.1016/j.idc.2015.02.009

8. Allerdice MEJ, Pritt BS, Sloan LM, Paddock CD, Karpathy SE. A real-time PCR assay for detection of the Ehrlichia muris-like agent, a newly recognized pathogen of humans in the upper Midwestern United States. Ticks Tick Borne Dis. 2016;7:146-9. http://dx.doi.org/10.1016/j.ttbdis.2015.10.004

9. Rand PW, Lacombe EH, Dearborn R, Cahill B, Elias S, Lubelczyk CB, et al. Passive surveillance in Maine, an area emergent for tick-borne diseases. J Med Entomol. 2007;44:111829. http://dx.doi.org/10.1093/jmedent/44.6.1118

Address for correspondence: Guang Xu, University of MassachusettsAmherst, Microbiology Fernald Hall, Rm B1, 270 Stockbridge Rd, Amherst, MA 01003,USA; email: gxu@umass.edu 